\title{
A decision support system for primary headache developed through machine learning
}

\author{
Fangfang Liu ${ }^{1}$, Guanshui Bao ${ }^{\text {Corresp., }}{ }^{1}$, Mengxia Yan $^{1}$, Guiming Lin ${ }^{1}$ \\ ${ }^{1}$ Shanghai Jiao Tong University School of Medicine, Shanghai Ninth People's Hospital, Shanghai, Huangpuqu, China \\ Corresponding Author: Guanshui Bao \\ Email address: baogs@163.com
}

Background $\square$ Primary headache is a disorder with a high incidence and low diagnostic accuracy; the incidence of migraine and tension-type headache ranks first among primary headaches. Artificial intelligence (Al) decision support systems have shown great potential in the medical field. Therefore, we attempt to use machine learning to build a clinical decision-making system for primary headaches.Methods $\square$ The demographic data and headache characteristics of 173 patients were collected by questionnaires. Decision tree, random forest, gradient boosting algorithm and support vector machine (SVM) models were used to construct a discriminant model and a confusion matrix was used to calculate the evaluation indicators of the models. Furthermore, we have carried out feature selection through univariate statistical analysis and machine learning.Results $\square$ In the models, the accuracy, F1 score were calculated through the confusion matrix. The logistic regression model has the best discrimination effect, with the accuracy reaching 0.84 and the area under the ROC curve also being the largest at 0.90. Furthermore, we identified the most important factors for distinguishing the two disorders through statistical analysis and machine learning: nausea/vomiting and photophobia/phonophobia. These two factors representpotential independent factors for the identification of migraines and tension-type headaches $\square$ with the accuracy reaching 0.74 and the area under the ROC curve being at 0.74 . Conclusions $\square$ Applying machine learning to the decision-making system for primary headaches can achieve a high diagnostic accuracy. Among them, the discrimination effect obtained by the integrated algorithm is significantly better than that of a single learner. Second, nausea/vomiting, photophobia/phonophobia may be the most important factors for distinguishing migraine from tension-type headaches. 
1

2

G।

Ye

\section{through machine learning}

4 Fangfang Liu ${ }^{1}$, Guanshui Bao ${ }^{1 *}$, Mengxia Yan ${ }^{1}$, Guiming Lin ${ }^{1}$

$5{ }^{1}$ Department of Neurology, Shanghai Ninth People's Hospital, Shanghai Jiao Tong University

6 School of Medicine, Shanghai, China

7 Corresponding Author: Guanshui Bao

8 Street Address, Shanghai, Huangpuqu, 200001, China

$9 \quad$ Email address: baogs@163.com

10

Background : Primary headache is a disorder with a high incidence and low diagnostic

accuracy; the incidence of migraine and tension-type headache ranks first among primary

headaches. Artificial intelligence (AI) decision support systems have shown great potential in the medical field. Therefore, we attempt to use machine learning to build a clinical decision-making system for primary headaches.

Methods : The demographic data and headache characteristics of 173 patients were collected by questionnaires. Decision tree, random forest, gradient boosting algorithm and support vector machine (SVM) models were used to construct a discriminant model and a confusion matrix was used to calculate the evaluation indicators of the models. Furthermore, we have carried out feature selection through univariate statistical analysis and machine learning.

Results : In the models, the accuracy, F1 score were calculated through the confusion matrix.

The logistic regression model has the best discrimination effect, with the accuracy reaching 0.84 and the area under the ROC curve also being the largest at 0.90 . Furthermore, we identified the most important factors for distinguishing the two disorders through statistical analysis and 
26 machine learning: nausea/vomiting and photophobia/phonophobia. These two factors represent

27 potential independent factors for the identification of migraines and tension-type headaches,

28 with the accuracy reaching 0.74 and the area under the ROC curve being at 0.74 . Conclusions

29 : Applying machine learning to the decision-making system for primary headaches can achieve

30 a high diagnostic accuracy. Among them, the discrimination effect obtained by the integrated

31 algorithm is significantly better than that of a single learner. Second, nausea/vomiting,

32 photophobia/phonophobia may be the most important factors for distinguishing migraine from

33 tension-type headaches.

34 Introduction

35 Headache is one of the most common symptoms in neurology clinics. More than $90 \%$ of the general population reports suffering from headache during any given year, which can be regarded as a lifetime history of head pain [1]. In China, the 1-year prevalence of primary headache is reported to be $23.8 \%$. The prevalence of migraine was $9.3 \%$, and that of tension-type headaches was $10.3 \%$ [2]. Due to the massive population base, patients spend 672.7 billion yuan each year - because of primary headaches, accounting for $2.24 \%$ of China's GDP [3]. Although headaches do not seriously threaten the lives of patients, they can severely affect their work and quality of life, cause them to withdraw from society, and place heavy burdens on the patients' psychology, 43 physiology and the families of patients as well as China's national economy [4-6].

44 Headaches are divided into primary headaches and secondary headaches. There are many causes 45 of headaches. Due to the similarity of symptoms, it is easy for general practitioners to miss or 46 misdiagnose the type of headache. Furthermore, the International Headache Society (IHS) released 47 the latest headache classification in January 2018, which is the International Classification of 48 Headache Disorders (ICHD-III) [7], which lists more than 200 headache variants. This 
49 complicated classification creates a very challenging task for general clinicians. There is no 50 objective gold standard, which contributes to the difficulty of diagnosing and classifying 51 headaches. In addition, because the medical community has generally not paid enough attention to 52 headaches in clinical practice for a long time, the proficiency level of clinicians regarding the 53 headache classification is uneven. For example, "vascular headache" and "nervous headache" are 54 still used to diagnose primary headache.

55 Thus, much progress remains to be made toward standardizing and improving the accuracy of the 56 clinical diagnosis of headache.

57 According to reports, primary headaches occur more frequently than secondary headaches, and the 58 incidence of migraine and tension-type headache ranks first among the types of primary headache 59 [8]. Migraines include migraines with aura and migraines without aura. Migraines without aura 60 are typically unilateral, pulsating, and moderate to severe headaches; daily physical activity can 61 exacerbate these headaches, and they are often accompanied by nausea/vomiting and/or 62 photophobia/phonophobia. Aura is the gradual appearance of visual, sensory, or other central 63 nervous system symptoms before or during the headache. Tension-type headaches are the most 64 common type of primary headache; attacks of this type of headache are not frequent and usually 65 last several minutes to several days. These headaches are typically characterized by mild to 66 moderate bilateral compression or band-like sensation; they are not aggravated by daily physical 67 activity and are not often accompanied by nausea/vomiting, or photophobia/phonophobia. 68 Although there are large differences between typical migraines and tension-type headaches, the 69 symptoms of most patients are not typical, especially in cases of tension-type headache and 70 migraine without aura. Thus, it is often difficult to distinguish between them. Due to the many 
71 differences in the treatment of the two disorders, misdiagnosis and missed diagnosis inevitably

72 delay the appropriate treatment of the patients[9].

73 At present, the development of Artificial Intelligence (AI) is in full swing. Automatic classifiers,

74 which are faster than clinicians due to their ability to analyze massive amounts of medical data,

75 can minimize errors in disease recognition and improve diagnostic accuracy. Support vector

76 machine (SVM) models, random forests, etc. have been used in the diagnosis of heart disease [10],

77 breast cancer [11], prostate cancer [12], Alzheimer's disease [13], and many other diseases. The

78 future of AI in neurology is promising, with potential applications ranging from the prediction of

79 outcomes of seizure disorder [14], the grading of brain tumors [15], the upskilling of neurosurgical

80 procedures [16], and the rehabilitation of stroke patients to the use of smartphone apps for

81 monitoring patient symptoms and progress [17].

82 For the proper recognition of headache, high-quality computer software could be very useful. As

83 early as 2013, Bartosz et al proposed the automatic diagnosis of primary headaches through

84 machine learning. The comparison of diagnostic performance between the advanced machine

85 learning technology and clinicians revealed that the computer decision support system achieved a

86 higher diagnostic accuracy [18]. More recently, Gilles et al proposed an end-to-end decision

87 support system to improve the efficiency of diagnosis and follow-up in the treatment of primary

88 headaches. The decision support system includes three large components and a shared backend: a

89 mobile application for patients, a web application for doctors to visualize the collected data, and

90 an automatic diagnosis module. In the automatic diagnosis module, a decision tree is used for

91 modeling [19]. Yin et al proposed a primary headache decision-making system based on

92 international headache diagnostic criteria and conducted a four-month clinical evaluation at the

93 International Headache Center of a tertiary hospital in Beijing. Good results have been obtained 
94 in terms of the sensitivity and specificity of this system for diagnosing headaches [20].

95 Considering the incomplete language rules when human experts express their knowledge,

96 Monire et al improved the algorithm and used the Learning-From-Examples (LEF) algorithm to

97 train the diagnostic fuzzy system, and the correct recognition rate reached $85 \%$. They further

98 proposed SVM- and multilayer perceptron (MLP)--based decision support systems, which

99 achieved accuracy rates of $90 \%$ and $88 \%$, respectively [21]. Simi'c et al create a hybrid

100 intelligent system for diagnosing primary headache disorders, applying various mathematical,

101 statistical and artificial intelligence techniques[22]. Although various types of research have been

102 devoted to computer decision support systems, there are still major obstacles to their widespread

103 use in clinical practice. Machine learning applied to medical records can be an effective tool to

104 predict disease. In China, machine learning methods for diagnosing primary headache remain

105 lacking.

106 Therefore, to achieve a higher headache diagnostic accuracy, we collected information from

107 primary headache patients in neurology clinics through questionnaires and then entered the data

108 into the system. We compared various machine learning algorithms to identify the best model.

109 Furthermore, through feature selection, we identified the most important factors for distinguishing

110 migraines from tension-type headaches, which provide a basis for clinicians to quickly diagnose

111 headaches.

\section{Materials \& Methods}

113 This is a cross-sectional study designed to obtain a diagnostic discriminant model for migraines

114 and tension-type headaches and to screen out the most important factors for distinguishing the two.

115 The study was approved by the Ethics Committee of the Ninth People's Hospital affiliated to 116 Shanghai Jiao Tong University Medicine (approval no.SH9H-2021-T72-1), and met the 
117 requirements of the Declaration of Helsinki. Eligible patients were patients diagnosed with

118 headaches between October 2019 and September 2020 at the Department of Neurology, Shanghai

119 Ninth People's Hospital. All the patients were residents of China. Before the study, we obtained

120 signed informed consent from the participating patients. Two weeks after a patient's questionnaire

121 was collected, we followed up on the patient's headache improvement to further verify the

122 diagnosis. Finally, we included 173 patients with a definite diagnosis of primary headache (84

123 patients with migraine headaches and 89 patients with tension-type headaches) for research.

\section{Data acquisition}

125 First, we designed a paper questionnaire for the outpatients to complete. The questionnaire included a total of 19 questions to collect the demographic data (age, sex, occupation, height, and weight) on the patients and their headache characteristics (course, duration, nature, location, severe the headache). After analysis and modification of the questionnaire by three experienced neurologists, the questionnaire was deemed effective for collecting patient-related information, and the data obtained were reliable to a certain extent.

132 Furthermore, information on related examinations and MRI were used to rule out the patient's secondary factors. Three neurologists were invited to make a diagnosis for each patient based on the questionnaire information we collected. Based on both the diagnosis and the follow-up results, each patient was accurately diagnosed. Due to the low proportion of primary headaches such as neuralgia and cluster headaches among the collected observations, we excluded these rare types of headaches to reduce the problems caused by sample imbalance. Ultimately, 173 patients (84 patients with migraines and 89 patients with tension-type headaches) were included in the study 139 (Fig1). Each patient's headache may have had multiple natures or been accompanied by multiple 
140 symptoms. Therefore, we performed a binary classification of the collected data and obtained a

141 total of 48 variables. Considering that the incidence of many variables was extremely low, we first

142 identified 10 variables with statistically significant differences between migraines and tension-

143 type headaches. After data transformation and data reduction, the data sheet used to acquire data

144 during the clinical interview is shown in Table 1.

\section{Discriminant model establishment}

146 Using the above 10 feature variables, we randomly divided the entire dataset into a training set

147 and a test set at several ratio variations $(60: 40,70: 30,80: 20)$ and used holdout and cross-

148 validation methods to build the primary headache discriminant models. Data analysis was

149 performed in Python (version 3.6.1). We used the decision tree, random forest, gradient boosting,

150 logistic regression, and SVM algorithms to construct discriminant models.

151 Decision tree

152 Decision tree is a nonparametric supervised learning method. The basic idea is to separate binary

153 variables and construct a tree that can be used to predict the category of new variables. It

154 traverses the training data and condenses the information into the internal nodes and leaf nodes.

155 Firstly, it summarizes decision rules from a series of data with features and labels, then present

156 these rules in a tree structure to solve classification problems.

157 Random forest

158 Random forest is an integrated algorithm that completes the learning task by constructing and

159 combining multiple learners. These learners are always classification trees. Firstly, the data is

160 classified by all trees, then the new category is determined by the majority decision principle. It

161 is nonparametrically interpretable and compatible with many types of data, with high prediction

162 accuracy. 
163 SVM

164 SVM is a binary supervised classification method, which shows many unique advantages in

165 solving small sample, nonlinear and high-dimensional pattern problems. The purpose of this

166 method is to find an optimal decision boundary in a multidimensional space, which can

167 maximize the distance between two closest points in different categories. This method can

168 process various types of data. From an academic point of view, SVM may be the closest machine

169 learning algorithm to deep learning.

170 Gradient boosting

171 Gradient boosting is another integrated algorithm. Like random forest, it constructs multiple

172 learners and brings them together into a final summed prediction. The main advantage of this

173 method is that can process various types of data flexibly, including continuous values and

174 discrete values.

175 Logistic regression

176 Logistic regression is a supervised learning algorithm to solve the binary classification problem,

177 which is used to estimate the probability of a certain category. It also can process various types

178 of data.

179 Furthermore, we combined the accuracy and F1 score as evaluation indicators of the model

180 through the common confusion matrix, and then measured the prediction result (receiver operating

181 characteristic, ROC) curve and the area under the ROC curve. The F1 score is the harmonic mean

182 of the precision and recall. It is used in statistics to measure the accuracy of two classifications and 183 assume that recall and precision are equally important.

$$
F 1 \text { score }=\frac{2 \text { Precision } * \text { Recall }}{\text { Precision }+ \text { Recall }}
$$

185 Feature selection 
186 The ten variables have redundancies in terms of allowing clinicians to quickly distinguish whether 187 a headache is a migraine or tension-type headache. Therefore, we identified the two variables that 188 are most meaningful for diagnosing migraines and tension-type headaches through feature ranking. 189 First, we adopted traditional univariate biometric analysis and then performed machine learning 190 analysis. For the univariate test, we used the Pearson correlation coefficient (PCC) [20], and the 191 chi-square test to compare each feature between the two groups. The PCC represents the linear 192 correlation between the elements of the two lists. If the elements in the two lists are linearly 193 correlated, the absolute value of the PCC will produce a high value close to 1; otherwise, it will be 194 close to 0 . The chi-square test is applied to two features to observe the probability of the 195 distribution occurring by chance. Each feature tested will produce a p-value. Although the P-value 196 does not represent the strength of the relationship between the two variables, it provides an 197 indication: the lower the p-value is, the greater certainty that the two variables are related. 198 Furthermore, we ranked the feature importance with the random forest method. The random forest 199 model is a nonlinear decision tree combination model. It is easy to implement and has superior 200 performance. It was once known as "the method that represents the level of integrated learning 201 technology". Using the random forest algorithm for feature selection is superior to the use of 202 linear discriminant analysis and mean squared error methods for eliminating redundant features. 203 The main idea is to judge how much each feature contributes to each tree in the random forest and 204 then to take the average value and evaluate the contribution of each feature separately. Compared 205 with the PCC, the random forest is more capable of mining the deep correlation of data features.

206 Afterwards, in a similar way we did before, we decided to investigate how the predictive power 207 would behave when using only the two selected features.

\section{Results}




\section{Patient baseline characteristics}

210 In our study, we enrolled 300 patients with primary headache. A total of 103 patients were 211 excluded according to the exclusion criteria. In addition, 24 patients were not followed up within

2122 weeks (Fig 1). Finally, we included 173 patients (84 patients with migraines and 89 patients with 213 tension-type headaches). We randomly divided the data from these 173 patients into a training set 214 and test set at several ratio variations (60:40, 70:30, 80:20). Our questionnaire collected 215 information on 48 patient characteristics through 19 questions. We used the chi-square test to 216 identify 10 informative characteristics and included them in the study (Table 1).

\section{Model building}

218 For the above 10 feature variables, we used the decision tree, random forest, gradient boosting, 219 logistic regression, and SVM algorithms to construct the discriminant models. After the cross220 validation,the mean accuracy, F1 score were calculated through the confusion matrix (Table 2), 221 the discrimination result curve (ROC curve) was constructed, and the area under the ROC curve 222 were measured. The mean accuracy of the decision tree is 0.72 , which was significantly lower 223 than that of the integrated learning algorithm and SVM models. The random forest, gradient 224 boosting algorithm, and SVM models have similar discrimination effects; their mean accuracy 225 scores were $0.80,0.79$, and 0.82 , and the mean areas under the ROC curves were $0.85,0.82$, and 2260.82 , respectively and the mean F1 score were $0.79,0.79$, and 0.81 , respectively. Logistic 227 regression had the best discrimination effect, with the mean accuracy reaching 0.84 and the mean 228 area under the ROC curve also being the largest among the methods, at 0.90 . The discrimination 229 effect achieved by the integrated algorithm was better than that of a single learner method, and 230 among the models, logistic regression achieved the best discrimination effect.

\section{Feature selection}


232 For feature selection, we applied two methods: univariate statistical analysis and machine learning.

233 For the univariate test, we used the PCC (Fig 2) and the chi-square test (Table 3) to compare each

234 feature between the two groups and rank them according to p-value. Through the univariate chi-

235 square tests, we determined that the smallest $p$-values were obtained for the variables indicating

236 whether the headache was accompanied by nausea/vomiting and whether the headache was

237 accompanied by photophobia/phonophobia. These two variables have the greatest power in 238 distinguishing the two disorders. The PCC confirmed the strong correlation between elements of 239 the two lists. The odds ratios (ORs) for nausea/vomiting and photophobia/phonophobia were 0.4 , 240 and were higher than those of the other headache-ralated variables. Through a simple correlation 241 analysis, we observe that patients with nausea/vomiting or photophobia/phonophobia were more 242 likely to be diagnosed with migraine headache than tension-type headache. To confirm and explore 243 the deeper relationship between the two disorders, we obtained the feature importance rankings 244 through the random forest model (Table 4). Among the variables, nausea/vomiting and 245 photophobia/phonophobia had importance values of 0.1897 and 0.1573 , respectively, ranking 246 them as the top two variables. To verify the predictive power of nausea/vomiting and 247 photophobia/phonophobia, we trained the logistic regression on these two features, with the mean 248 accuracy reaching 0.74 and the mean area under the ROC curve reaching 0.74 ( Table 5).

249 In clinical practice, compared with patients with tension-type headaches, migraine patients have 250 more severe headaches and longer disease courses, and their headaches are usually accompanied 251 by nausea/vomiting and photophobia/phonophobia. In contrast, tension-type headaches are 252 generally mild, and not accompanied by nausea/vomiting and photophobia/phonophobia. Our 253 results are consistent with clinical experience. Therefore, we further compared the headache 254 severity and nausea/vomiting and photophobia/phonophobia between the two types of patients 
255 (Fig 3). Compared with patients with tension-type headaches, migraine patients were more likely

256 to experience nausea/ vomiting and photophobia/phonophobia. Migraines were more severe and

257 were mainly distributed among the moderate to severe cases, while tension-type headaches were

258 mainly distributed among the mild to moderate cases.

\section{Discussion}

\section{Model building}

261 AI is being applied to all types of fields, and its application to the medical field is a way for us to 262 follow this trend. We used machine learning to identify primary headaches, which provided a 263 starting point for advancing the transformation of AI. In this study, we established a discriminant 264 model for the two types of primary headaches (migraine and tension-type headache) by machine 265 learning algorithms based on 10 indicators. The diagnosis of primary headache, which is a 266 functional disorder without an objective gold standard for diagnosis, is very difficult. Especially 267 for the intermediate state of these two diseases, the ICHD-III diagnostic criteria are suitable for 268 the diagnosis of only typical headache. For atypical headache and the intermediate headache state, 269 many clinicians can rely only on their own clinical experience, and this subjective approach 270 inevitably has a great impact on the accuracy of disease diagnosis. In other words, clinical 271 diagnoses made by clinicians are highly subjective, varied and inconsistent. Furthermore, some 272 scholars believe that there may be overlap of multiple primary headaches, where multiple headache 273 symptoms exist simultaneously. Such overlapping headaches are common in cases of migraine 274 and tension-type headache. In addition, there are treatment differences among the different types 275 of headaches. Only clear diagnoses can improve these treatments. This intermediate headache state 276 and the overlapping conditions make it difficult for clinicians to accurately diagnose primary 277 headaches. Previous studies on primary headaches have been focused mainly on expert decision- 
278 making systems based on international diagnostic standards [23-25]. However, it is difficult to

279 make a diagnosis based on the ICHD-III criteria for the intermediate state and the overlap of

280 clinical diseases. Perhaps it would be more efficient and effective to diagnose diseases through

281 individualized learning and reasoning based on samples than via a pure expert decision-making

282 system. Machine learning methods are an attractive option for such a task because they offer fast,

283 precise and intelligent analysis of multidimensional data. Therefore, in this study, we constructed

284 a model through different machine learning algorithms and explore the differences between

285 samples. In addition, for related headache data, it is possible to perform cluster analysis and

286 improve headache classification. Because of the subjective nature of the diagnosis, perform their

287 evaluations independently and reach different conclusions for the same case. After the promotion

288 and application of the decision-making system and through continuous learning and revision, the

289 diagnostic criteria used by clinicians can develop in the same direction.

\section{Feature selection}

291 To help clinicians quickly grasp the focus of the disease, the 10 variables were screened through

292 univariate statistical analysis and machine learning to identify the most important factors for

293 distinguishing migraines and tension-type headaches. The two most important factors were

294 nausea/vomiting and photophobia/phonophobia. They represent potential independent predictors.

295 In previous studies on simplified headache diagnostic criteria [26], a univariate migraine model

296 including nausea achieved a positive likelihood ratio of 4.8 and a negative likelihood ratio of 0.23.

297 By including the three variables for nausea, photophobia, and throbbing headache, the migraine

298 model achieved a positive likelihood ratio of 6.7 and a negative likelihood ratio of 0.23 . The ID

299 Migraine $^{\mathrm{TM}}$ screening instrument has been found to be an effective and reliable migraine screening

300 instrument, among the possible variables, disability, nausea, and photophobia provide the best 
301 performance [27]. In our research, although we did not separately screen for nausea, vomiting, 302 photophobia, and phonophobia, the results we obtained through statistical analysis and machine

303 learning are generally consistent with those of previous studies. To ensure the integrity of the

304 experiment, we trained the logistic regression based on these two features. According to the results,

305 the multi-features model is better than the two-features model. However, the two features selected

306 can help clinicians grasp the focus of the disease as soon as possible. Nausea/vomiting,

307 photophobia/phonophobia, and phonophobia play a vital role in distinguishing migraines from 308 tension-type headaches.

309 Inevitably, our study has flaws. First, our discriminant model includes only the two types of

310 headaches with the highest incidence: migraine and tension-type headache. Although the model

311 can solve most of the problems related to the clinical diagnosis of headaches, other primary

312 headaches and secondary headaches are not included. Therefore, adding other headache categories

313 will be a future direction of expansion of our system. Second, the diagnosis of headache is

314 strongly affected by the clinical experience of the clinician. Although we followed up with each

315 patient after 2 weeks to assess headache improvement and verify the diagnosis, changes in the

316 patient's living habits or other factors might have impacted on the follow-up results. Third, we

317 included headache patients who visited a doctor, leading to selection bias. Patients with mild

318 headaches who did not seek medical attention from a doctor were not included in the study. Finally,

319 our sample size was small, we need to increase the sample size to verify and test the model.

320 Conclusions

321 Primary headache is a disorder with high incidence and low diagnostic accuracy. The goal of this

322 research is focused on the design and implementation of decision support system for diagnosing

323 primary headaches. This study used machine learning to construct a discriminant model for

324 migraines and tension-type headaches. The discriminant effect achieved by the integrated 
325 algorithms, such as the random forest and gradient boosting algorithms, was better than that of a

326 single learner approaches, and the logistic regression model achieved the best discrimination

327 effect. Further research could be focused on creating new and more efficient tools and systems to

328 help and improve physicians' work and make diagnoses better. In addition, we identified the most

329 important factors for the identification of the two diseases through statistical analysis and machine

330 learning: nausea/vomiting and photophobia/phonophobia. These two factors represent potential

331 independent factors for identifying migraines and tension-type headaches, which can help

332 clinicians quickly grasp the focus of headaches. However, our sample size was small, and we

333 need to increase the sample size to verify and improve the model.

334 Acknowledgements

335 Funding from the Jinhua Science and Technology Bureau (No.2020-3-036) and the project of the

336 Shanghai Science and Technology Commission (14411972200) are gratefully acknowledged. X-

337 MY and M-GL collected the data, and contributed to the data management. F-FL conceived the

338 study, compared the results of the biostatistics feature rankings and the machine learning feature

339 rankings, wrote the manuscript. W-WA, G-SB, and F-JL helped design and revise the

340 questionnaire. G-SB revised the final version of the manuscript. All authors have read and

341 approved the final manuscript.

342

343

344

345 References

346 [1] K. Hagen, A. N. Åsberg, B. L. Uhlig, E. Tronvik, E. Brenner, M. Stjern, G. Helde, G. B.

347 Gravdahl and T. Sand. 2018. The epidemiology of headache disorders: a face-to-face interview of 348 participants in HUNT4. J Headache Pain 19:25. 10.1186/s10194-018-0854-2

349 [2] S. Yu, R. Liu, G. Zhao, X. Yang, X. Qiao, J. Feng, Y. Fang, X. Cao, M. He and T. Steiner.

350 2012. The prevalence and burden of primary headaches in China: a population-based door-to-door

351 survey. Headache 52:582-591. 10.1111/j.1526-4610.2011.02061.x 
352 [3] C. Yao, Y. Wang, L. Wang, Y. Liu, J. Liu, J. Qi, Y. Lin, P. Yin and M. Zhou. 2019. Burden of 353 headache disorders in China, 1990-2017: findings from the Global Burden of Disease Study 2017. 354 J Headache Pain 20:102. 10.1186/s10194-019-1048-2

355 [4] T. Takeshima, Q. Wan, Y. Zhang, M. Komori, S. Stretton, N. Rajan, T. Treuer and K. Ueda. 356 2019. Prevalence, burden, and clinical management of migraine in China, Japan, and South Korea:

[5] D. Saylor and T. J. Steiner. 2018. The Global Burden of Headache. Semin Neurol 38:182-190. $10.1055 / \mathrm{s}-0038-1646946$

[6] K. Malmberg-Ceder, M. Haanpää, P. E. Korhonen, H. Kautiainen, V. Veromaa and S. Soinila. 2019. The role of psychosocial risk factors in the burden of headache. J Pain Res 12:1733-1741. $10.2147 / \mathrm{jpr} . \mathrm{S} 165263$

[7] 2018. Headache Classification Committee of the International Headache Society (IHS) The International Classification of Headache Disorders, 3rd edition. Cephalalgia 38:1-211. $10.1177 / 0333102417738202$

[8] Á. L. G. MD, E. R. MD, S. H. MD, M. J. N. NP, L. B. NP, M. L. P. MD, E. C. MD, P. M. MD, R. F. MD and PhD. 2011. Characteristics of the First 1000 Headaches in an Outpatient Headache Clinic Registry. Headache: The Journal of Head and Face Pain 51.

[9] J. K. Porter, G. L. Di Tanna, R. B. Lipton, S. Sapra and G. Villa. 2019. Costs of Acute Headache Medication Use and Productivity Losses Among Patients with Migraine: Insights from Three Randomized Controlled Trials. Pharmacoecon Open 3:411-417. 10.1007/s41669-018-0105-0 [10] C. Krittanawong, H. U. H. Virk, S. Bangalore, Z. Wang, K. W. Johnson, R. Pinotti, H. Zhang, S. Kaplin, B. Narasimhan, T. Kitai, U. Baber, J. L. Halperin and W. H. W. Tang. 2020. Machine learning prediction in cardiovascular diseases: a meta-analysis. Sci Rep 10:16057. $10.1038 / \mathrm{s} 41598-020-72685-1$

[11] M. W. Huang, C. W. Chen, W. C. Lin, S. W. Ke and C. F. Tsai. 2017. SVM and SVM Ensembles in Breast Cancer Prediction. PLoS One 12:e0161501. 10.1371/journal.pone.0161501 [12] J. Li, Z. Weng, H. Xu, Z. Zhang, H. Miao, W. Chen, Z. Liu, X. Zhang, M. Wang, X. Xu and Q. Ye. 2018. Support Vector Machines (SVM) classification of prostate cancer Gleason score in central gland using multiparametric magnetic resonance images: A cross-validated study. Eur J Radiol 98:61-67. 10.1016/j.ejrad.2017.11.001

[13] T. Shen, J. Jiang, Y. Li, P. Wu, C. Zuo and Z. Yan. 2018. Decision Supporting Model for One-year Conversion Probability from MCI to AD using CNN and SVM. Annu Int Conf IEEE Eng Med Biol Soc 2018:738-741. 10.1109/EMBC.2018.8512398

[14] B. Abbasi and D. M. Goldenholz. 2019. Machine learning applications in epilepsy. Epilepsia 60:2037-2047. 10.1111/epi.16333

[15] M. Kocher, M. I. Ruge, N. Galldiks and P. Lohmann. 2020. Applications of radiomics and machine learning for radiotherapy of malignant brain tumors. Strahlenther Onkol 196:856-867. 10.1007/s00066-020-01626-8

[16] J. T. Senders, O. Arnaout, A. V. Karhade, H. H. Dasenbrock, W. B. Gormley, M. L. Broekman and T. R. Smith. 2018. Natural and Artificial Intelligence in Neurosurgery: A Systematic Review. Neurosurgery 83:181-192. 10.1093/neuros/nyx384

[17] S. H. Chae, Y. Kim, K. S. Lee and H. S. Park. 2020. Development and Clinical Evaluation of a Web-Based Upper Limb Home Rehabilitation System Using a Smartwatch and Machine Learning Model for Chronic Stroke Survivors: Prospective Comparative Study. JMIR Mhealth Uhealth 8:e17216. 10.2196/17216

[18] B. Krawczyk, D. Simić, S. Simić and M. Woźniak. 2013. Automatic diagnosis of primary 
398

399

400

401

402

403

404

405

406

407

408

409

410

411

412

413

414

415

416

417

418

419

420

421

422

423

424

425

426 headaches by machine learning methods. Open Medicine 8:157-165. 10.2478/s11536-012-0098-5 [19] G. Vandewiele, F. De Backere, K. Lannoye, M. Vanden Berghe, O. Janssens, S. Van Hoecke, V. Keereman, K. Paemeleire, F. Ongenae and F. De Turck. 2018. A decision support system to follow up and diagnose primary headache patients using semantically enriched data. BMC Med Inform Decis Mak 18:98. 10.1186/s12911-018-0679-6

[20] Y. Z. D. Z. K. Xiangyong. 2019. Assistant decision-making system based on international diagnostic criteria for primary headache disorders. Application Research of Computers 36:2.

[21] M. Khayamnia, M. Yazdchi, A. Heidari and M. Foroughipour. 2019. Diagnosis of Common Headaches Using Hybrid Expert-Based Systems. J Med Signals Sens 9:174-180. 10.4103/jmss.JMSS_47_18

[22] S. Simić, J. R. Villar, J. L. Calvo-Rolle, S. R. Sekulić, S. D. Simić and D. Simić. 2021. An Application of a Hybrid Intelligent System for Diagnosing Primary Headaches. Int J Environ Res Public Health 18. 10.3390/ijerph18041890

[23] R. Costabile, G. Catalano, B. Cuteri, M. C. Morelli, N. Leone and M. Manna. 2020. A logicbased decision support system for the diagnosis of headache disorders according to the ICHD-3 international classification.

[24] A. Roesch, M. A. Dahlem, L. Neeb and T. Kurth. 2020. Validation of an algorithm for automated classification of migraine and tension-type headache attacks in an electronic headache diary. J Headache Pain 21:75. 10.1186/s10194-020-01139-w

[25] L. Hui, H. C. Keh, C. C. Meng and Z. Y. Liu. 2018. Clinical Application of Decision Support System for Treatment of Migraine. International Conference on Frontier Computing.

[26] V. T. Martin, D. B. Penzien, T. T. Houle, M. E. Andrew and K. R. Lofland. 2005. The predictive value of abbreviated migraine diagnostic criteria. Headache 45:1102-1112. 10.1111/j.1526-4610.2005.00234.x

[27] R. B. Lipton, D. Dodick, R. Sadovsky, K. Kolodner, J. Endicott, J. Hettiarachchi and W. Harrison. 2003. A self-administered screener for migraine in primary care: The ID Migraine validation study. Neurology 61:375-382. 10.1212/01.wnl.0000078940.53438.83 
Table $\mathbf{1}$ (on next page)

Patient baseline characteristics 
Table 1 Patient baseline characteristics

\begin{tabular}{|c|c|c|c|c|}
\hline Characteristics & $\begin{array}{l}\text { Migraine } \\
(n=84)\end{array}$ & $\begin{array}{l}\text { Tension-type } \\
\text { headache }(\mathrm{n}=89)\end{array}$ & Total & $\mathrm{P}-$ value \\
\hline $\operatorname{Sex} / \mathrm{n}(\%)$ & - & - & - & - \\
\hline Female & $20(23.8)$ & $39(43.8)$ & $59(34.1)$ & $\mathrm{P}=0.01$ \\
\hline Male & $64(76.2)$ & $50(56.2)$ & $114(65.9)$ & \\
\hline \multicolumn{5}{|l|}{ Course/n(\%) } \\
\hline Year & $11(13.1 \%)$ & $38(42.7 \%)$ & $49(28.3)$ & $\mathrm{P}<0.001$ \\
\hline Month & $73(86.9 \%)$ & $51(57.3 \%)$ & $114(65.9)$ & \\
\hline \multicolumn{5}{|l|}{ Throbbing/n(\%) } \\
\hline Yes & $17(20.2)$ & $6(6.7)$ & $23(13.3)$ & $\mathrm{P}=0.01$ \\
\hline No & $67(79.8)$ & 83(93.3) & $150(86.7)$ & \\
\hline \multicolumn{5}{|l|}{ Occiput/n(\%) } \\
\hline Yes & $22(26.2)$ & $43(48.3)$ & $65(37.6)$ & $\mathrm{P}=0.00$ \\
\hline No & $62(73.8)$ & $46(51.7)$ & $108(62.4)$ & \\
\hline \multicolumn{5}{|c|}{ Severe intensity/n(\%) } \\
\hline Light & $13(15.5)$ & $30(33.7)$ & $43(24.9)$ & \\
\hline Medium & $44(52.4)$ & $51(57.3)$ & $95(54.9)$ & $\mathrm{P}<0.001$ \\
\hline Heavy & $27(32.1)$ & $8(9.0)$ & $35(20.2)$ & \\
\hline \multicolumn{5}{|c|}{ Nausea/ vomiting /n(\%) } \\
\hline Yes & $44(52.4)$ & $16(18.0)$ & $60(34.7)$ & $\mathrm{P}<0.001$ \\
\hline No & $40(47.6)$ & $73(82.0)$ & $113(65.3)$ & \\
\hline \multicolumn{5}{|c|}{ Photophobia/ phonophobia /n(\%) } \\
\hline Yes & $27(32.1)$ & $4(4.5)$ & 31(17.9) & $\mathrm{P}<0.001$ \\
\hline No & $57(67.9)$ & $85(95.5)$ & $142(82.1)$ & \\
\hline \multicolumn{5}{|l|}{ Spark/n(\%) } \\
\hline Yes & $11(13.1)$ & $3(3.4)$ & $14(8.1)$ & $\mathrm{P}=0.02$ \\
\hline No & $73(86.9)$ & $86(96.6)$ & 159(91.9) & \\
\hline \multicolumn{5}{|c|}{ Change after activities/n(\%) } \\
\hline Aggravate & $41(48.8)$ & $18(20.2)$ & $59(34.1)$ & \\
\hline Unchanged & $38(45.2)$ & $62(69.7)$ & $100(57.8)$ & $\mathrm{P}<0.001$ \\
\hline Relieve & $5(6.0)$ & $9(10.1)$ & $14(8.1)$ & \\
\hline \multicolumn{5}{|c|}{ Alleviative methods/n(\%) } \\
\hline Persistence & $9(10.7)$ & $14(15.7)$ & $23(13.3)$ & \\
\hline Rest & $25(29.8)$ & $45(50.6)$ & $70(40.5)$ & $\mathrm{P}=0.00$ \\
\hline Drug & $48(57.1)$ & $25(28.1)$ & $73(42.2)$ & \\
\hline Else & $2(2.4)$ & $5(5.6)$ & $7(4.0)$ & \\
\hline
\end{tabular}


Table 2 (on next page)

Evaluation of the discriminant effect of various models 
1

2

Table 2 Evaluation of the discriminant effect of various models

3

\begin{tabular}{cccccccccccccc}
\hline & \multicolumn{3}{c}{$80: 20$} & \multicolumn{1}{c}{70.30} & \multicolumn{3}{c}{$60: 40$} & \multicolumn{3}{c}{ Mean } \\
\cline { 2 - 12 } & Accuracy & F1 & AUC & Accuracy & F1 & AUC & Accuracy & F1 & AUC & Accuracy & F1 & AUC \\
\cline { 2 - 12 } & 0.74 & 0.69 & 0.74 & 0.74 & 0.65 & 0.64 & 0.64 & 0.69 & 0.78 & 0.72 & 0.68 & 0.72 \\
$\begin{array}{c}\text { Decision tree } \\
\text { Random } \\
\text { Forests }\end{array}$ & 0.89 & 0.86 & 0.90 & 0.90 & 0.78 & 0.79 & 0.79 & 0.74 & 0.85 & 0.80 & 0.79 & 0.85 \\
$\begin{array}{c}\text { Gradient } \\
\text { boosting }\end{array}$ & 0.89 & 0.87 & 0.91 & 0.91 & 0.71 & 0.70 & 0.70 & 0.79 & 0.86 & 0.79 & 0.79 & 0.82 \\
$\begin{array}{c}\text { Logistic } \\
\text { regression }\end{array}$ & 0.91 & 0.90 & 0.95 & 0.95 & 0.82 & 0.88 & 0.88 & 0.77 & 0.87 & 0.84 & 0.83 & 0.90 \\
SVM-linear & 0.89 & 0.87 & 0.84 & 0.84 & 0.81 & 0.82 & 0.82 & 0.75 & 0.81 & 0.82 & 0.81 & 0.82 \\
\hline
\end{tabular}

4 
Table 3 (on next page)

chi-square test 
1

2

Table 3 chi-square test

\begin{tabular}{cl}
\hline Characteristic variable & $\mathrm{P}$-value \\
\hline Photophobia/phonophobia & $\mathrm{P}<0.001$ \\
Nausea/vomiting & $\mathrm{P}<0.001$ \\
Course & $\mathrm{P}<0.001$ \\
Change after activities & $\mathrm{P}<0.001$ \\
Severe intensity & $\mathrm{P}<0.001$ \\
Alleviative way & $\mathrm{P}=0.00$ \\
Occiput & $\mathrm{P}=0.00$ \\
Throbbing & $\mathrm{P}=0.01$ \\
Spark & $\mathrm{P}=0.02$ \\
\hline
\end{tabular}

3 
Table 4 (on next page)

Random forest importance ranking 
1

2

3

4

西
Table 4 Random forest importance ranking

\begin{tabular}{cc}
\hline Characteristic variable & importance \\
\hline Nausea/vomiting & 0.1897 \\
Photophobia/phonophobia & 0.1573 \\
Change after activities & 0.1144 \\
Course & 0.1124 \\
Severe intensity & 0.1083 \\
Alleviative way & 0.0837 \\
Occiput & 0.0754 \\
Spark & 0.0604 \\
Throbbing & 0.0444 \\
\hline
\end{tabular}




\section{Table 5 (on next page)}

Evaluation of the predictive powert of the two selected features. 
Table 5 Evaluation of the predictive powert of the two selected features.

2

3

4

\begin{tabular}{cccc}
\hline Logistic regression & Accuracy & F1-score & ROC-AUC \\
\hline $80: 20$ & 0.74 & 0.61 & 0.71 \\
$70: 30$ & 0.71 & 0.69 & 0.73 \\
$60: 40$ & 0.76 & 0.74 & 0.78 \\
Mean & 0.74 & 0.68 & 0.74 \\
\hline
\end{tabular}


Figure 1

\section{Study flow chart}

300 headache datients

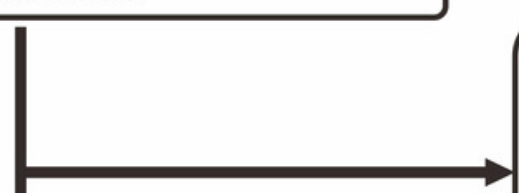

103 patients were excluded.

1) Neuralgia $(n=37)$

2) Low intracranial pressure headache $(n=3)$

3) Cluster headache $(n=5)$

4) Secondary headache $(n=58)$

197 patients with migraines and tension-type headaches were eligible

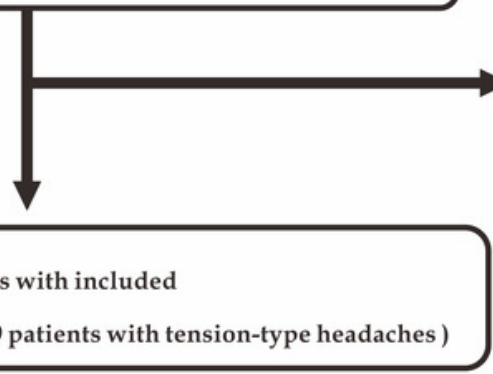

24 patients failed to follow up

173 patients with included

( 84 patients with migraines and 89 patients with tension-type headaches ) 
Figure 2

Pearson correlation coefficient

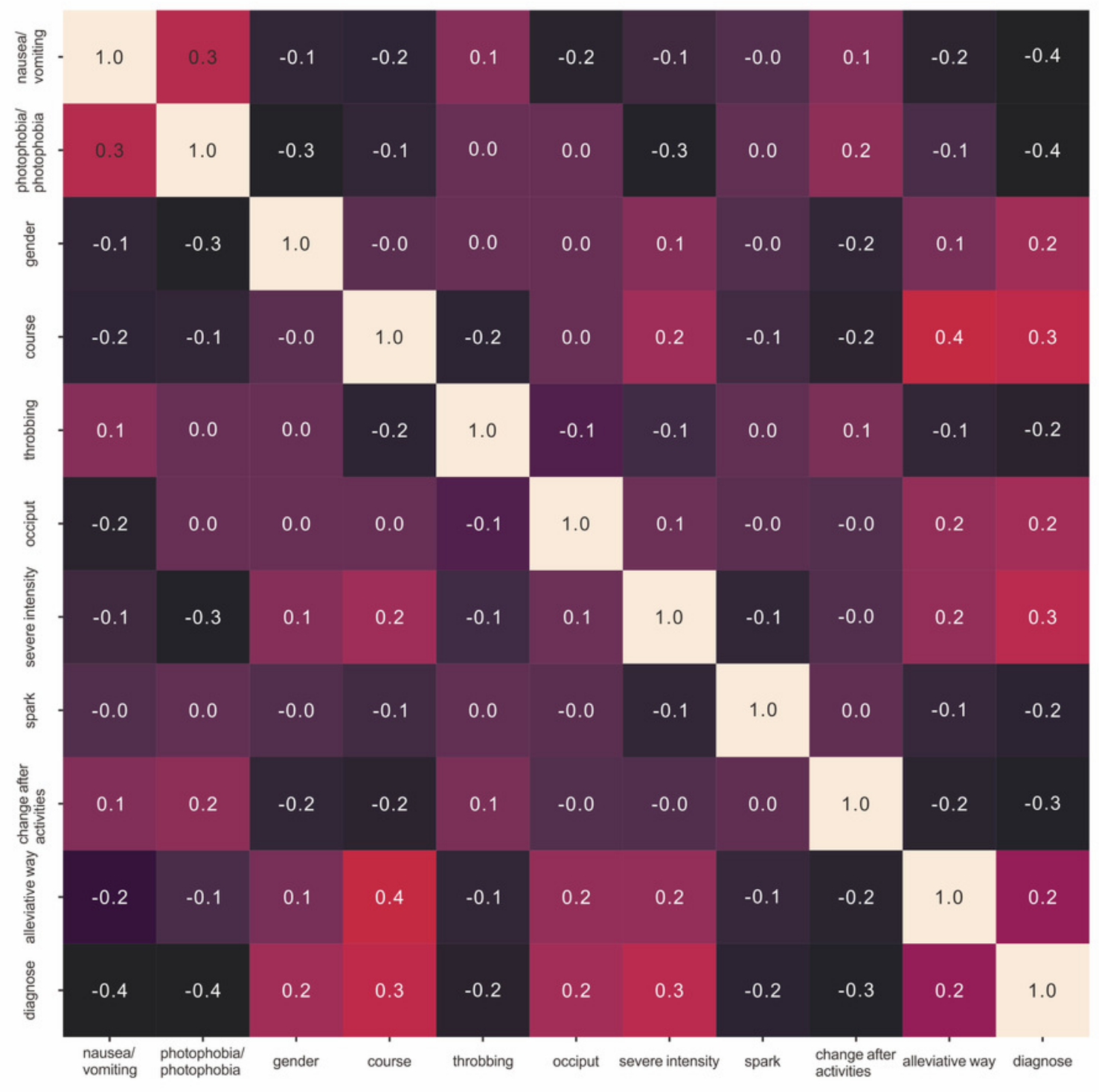


Figure 3

The correlation between headache severe intensity, nausea/vomiting, and photophobia/phonophobia
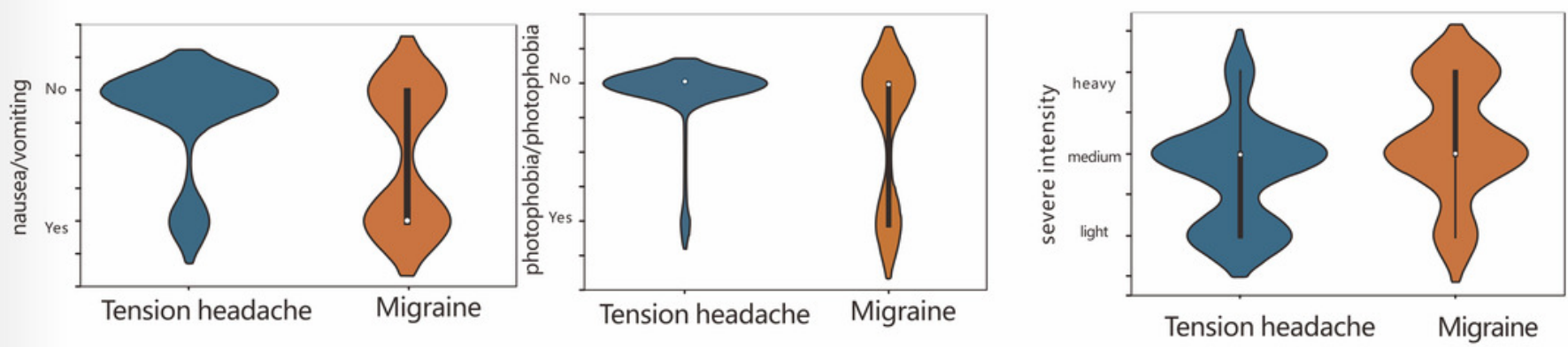